\title{
Consideraciones académicas y administrativas para implementación de Capstone en ingeniería civil: Estudio de caso
}

\author{
Academic and Administrative Issues for Implementation of Capstone Course in Civil \\ Engineering programs: Case Study
}

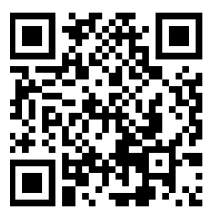

\author{
María Fernanda Serrano-Guzmán ${ }^{1}$ \\ Pontificia Universidad Javeriana Cali \\ Cali, Colombia \\ maria.serrano@javerianacali.edu.co \\ http://orcid.org/0000-0002-7366-6597 \\ Diego Darío Pérez-Ruiz² \\ Pontificia Universidad Javeriana Cali \\ Cali, Colombia \\ ddperez@javerianacali.edu.co \\ http://orcid.org/0000-0002-9656-2803 \\ Luz Marina Torrado-Gómez ${ }^{3}$ \\ Universidad Pontificia Bolivariana Seccional Bucaramanga \\ Bucaramanga, Colombia \\ luz.torrado@upb.edu.co \\ http://orcid.org/0000-0001-8941-7344 \\ Norma Cristina Solarte-Vanegas ${ }^{4}$ \\ Universidad Pontificia Bolivariana Seccional Bucaramanga \\ Bucaramanga, Colombia \\ norma.solarte@upb.edu.co \\ http://orcid.org/0000-0002-2392-3663
}

Recibido 9 de mayo de 2016 • Corregido 7 de abril de 2017 • Aceptado 25 de abril de 2017

\footnotetext{
${ }^{1}$ Docente de Ingeniería Civil en la Pontificia Universidad Javeriana de Cali. Doctora en Ingeniería Civil de la Universidad de Puerto Rico Recinto Universitario de Mayagüez, Magister en Ingeniería de la Universidad de Puerto Rico Recinto Universitario de Mayagüez, Especialista en Ingeniería Ambiental de la Universidad Pontifica Bolivariana Seccional Bucaramanga. Especialista en Gerencia de Proyectos de Construcción. Ingeniera Civil de la Universidad Industrial de Santander.

${ }^{2}$ Docente de Ingeniería Civil en la Pontificia Universidad Javeriana de Cali en Ingeniería Civil. Doctor en Ingeniería Civil de la Universidad de Texas en Arlington, Magister en Ingeniería de Tránsito y Transporte de la Universidad del Cauca. Magister en Ingeniería Civil énfasis en Recursos Hídricos de la Universidad de Puerto Rico Recinto Universitario de Mayagüez Ingeniero Civil de la Universidad del Cauca.

${ }^{3}$ Docente de Ingeniería Civil en la Universidad Pontificia Bolivariana Seccional Bucaramanga. Magister en Geotecnia de la Universidad Industrial de Santander. Especialista en Gerencia e Interventoría de Obras Civiles e Ingeniera Civil de la Universidad Pontificia Bolivariana Seccional Bucaramanga. Tecnóloga en Obras Civiles en la Universidad Francisco de Paula Santander. Subgerente de la empresa INGEAS SAS.

${ }^{4}$ Ingeniero Civil, M.Sc. en Tránsito y Transporte de la Universidad del Cauca, Especialista en Carreteras de la Universidad Nacional de San Juan Argentina. Docente de la Universidad Pontificia Bolivariana Seccional Bucaramanga. Directora de la Facultad de Ingeniería Civil.
} 
doi: http://dx.doi.org/10.15359/ree.21-2.17

URL: http://www.una.ac.cr/educare

CORREO: educare@una.cr

Resumen: El trabajo final de diseño ("Capstone" en inglés) considerado como requisito final de grado para estudiantes de ingeniería, busca incentivar el trabajo en grupo para encontrar la solución a una problemática relacionada con el ejercicio profesional, aplicando los conocimientos de diseño adquiridos durante el proceso de formación. Esta modalidad de trabajo final de grado exige una reestructuración académico-administrativa que garantice el resultado satisfactorio en el tiempo previsto para este propósito. El objetivo del presente trabajo es identificar la percepción del estudiantado de ingeniería civil de la Pontificia Universidad Javeriana de Cali en relación con el "Capstone" como trabajo de final de grado y partiendo de este análisis, plantear estrategias administrativas que favorezcan el desarrollo de los trabajos en esta modalidad. Para ello, se preparó un instrumento de consulta con la modalidad de encuesta en donde participaron 47 estudiantes. El instrumento diseñado permitió recoger información sobre las experiencias del aprendizaje basado en problemas en las diferentes asignaturas cursadas durante su formación académica, la composición y evaluación de los grupos de trabajo en los diferentes cursos y la autoevaluación del estudiantado frente al desempeño y participación en los grupos conformados. Un 87.2\% del estudiantado considera que el "Capstone" es una opción de grado adecuada; pero que tiene una gran dificultad en el manejo del rendimiento del grupo, ya que es común que el trabajo recaiga en una o dos personas. Aun así, $86.2 \%$ del estudiantado considera que la participación del grupo fue adecuada y el $95.2 \%$ considera que su desempeño fue bueno. Los anteriores hallazgos indican que se deben reforzar las estrategias de trabajo colaborativo y capacitar al personal docente-tutor del "Capstone" sobre estrategias de medición y evaluación del desempeño individual y grupal. Los resultados obtenidos serán de utilidad para el mejoramiento curricular y administrativo de las asignaturas asociadas al "Capstone" y podría ser de utilidad para aquellas universidades que consideren la implementación del "Capstone" como trabajo terminal de grado.

Palabas claves: Redacción de informe; requisito; subgraduado; trabajo colaborativo; Capstone.

Abstract: A capstone course is a culminating academic experience designed to encourage teamwork among students by applying their five years of classroom knowledge to solve realistic engineering problems. This emerging capstone experience demands academic and administrative changes to guarantee a satisfactory result within the time spared for this purpose. The main objective of this research is to assess students' perception regarding the capstone project as a culminating academic experience, and to use the analysis results to propose academic and administrative changes required to support and guarantee the successful achievement of this type of final degree project. To reach this goal, an assessment instrument was prepared and applied to 47 students enrolled in a capstone course. The instrument allowed collecting information regarding problem-based learning experience, teamwork experience, and self-perception concerning students' own behaviors and attitude during teamworking. On this regard, $87.2 \%$ of students considered capstone as a suitable final degree experience; however, it is challenging because it is known that student's participation during teamworking is uncommon and usually just two students are committed. In spite of this, $86.2 \%$ of students considered that teamwork was satisfactory and $95.2 \%$ of them recognized that their self-behavior and attitude during teamworking was good. Based on the results we recommend to strengthen teamwork strategies among students, and to instruct teachers about assessment strategies to evaluate individual and teamwork performance of students. It is expected that these findings can help improve the capstone curriculum related to courses and their administration. These findings are also supposed to serve as a previous experience to those universities committed to include capstone as a culminating degree experience.

Keywords: Technical writing; undergraduate; requirements; collaborative work; Capstone. 


\section{Introducción १}

En la actualidad, la industria y el medio laboral requieren profesionales con creatividad, capacidad de análisis; conocimiento técnico; habilidades para comunicarse, interactuar y tomar decisiones; espíritu de liderazgo (Oehrtman, Smolen, Hoblet \& Phillips, 2010; Shin, Lee, Ahn, \& Jung, 2013); capacidad para aplicar el pensamiento crítico-interpretativo (Good \& Mclntyre, 2015); pensamiento analítico, ético y con curiosidad intelectual (Vellurattil, Puglisi, Johnson, \& Slonek, 2014). Para responder a estas exigencias se deben proponer cambios que permitan ajustar los currículos y el estilo de enseñanza a los requerimientos de las empresas públicas o privadas que necesitan profesionales con diferentes habilidades (Blackford \& Shi, 2015).

La estrategia de implementación de un curso terminal de diseño ("Capstone") permite al estudiantado llenar el vacío que aparentemente existe entre el currículo y las habilidades y destrezas que demanda el ejercicio profesional en las empresas de ingeniería (Gruenther, Bailey, Wilson, Plucker \&Hashmi, 2009), mientras se pone en práctica el conocimiento adquirido durante el proceso de formación; es más, se puede decir que es una manera para lograr el acercamiento del estudiantado a su ejercicio profesional, incluso, para establecer las bases para la creación de su propia empresa (Odora, 2015). Usualmente, el curso "Capstone" se lleva a cabo en el último año de estudios, lo que ayuda a integrar el conocimiento de diseño adquirido durante el tiempo de formación (Quinot \& van Tonder, 2014). La Tabla 1 resume la percepción de algunos estudios en diferentes disciplinas respecto al "Capstone".

Como se observa, el "Capstone" permite la integración del currículo, brinda el espacio para replantear el aprendizaje y es considerado una transición al ambiente laboral (Stanley \& Marsden, 2013), da la oportunidad a que la persona futura profesional experimente la importancia y necesidad del trabajo colaborativo (Lubbers, 2011). Este curso es requisito para la acreditación de alta calidad de ABET (Accreditation Board for Engineering Education) (Agboola, Hashemipour, Egelioglu, Atikol, \& Hacisevki, 2012).

Aunque existen otras alternativas que buscan el acercamiento al ejercicio ocupacional, como es el caso de la práctica profesional, la cual tiene un tiempo corto de contacto con la empresa o la industria y se realiza de manera individual (Helms \& Whitesell, 2013), el "Capstone" puede desarrollarse en un lapso de dos semestres, tiempo durante el cual el estudiantado cuenta con la asesoría y apoyo permanente de profesionales con pericia en las distintas áreas involucradas en el proyecto de diseño seleccionado. Estos grupos profesionales guían al estudiantado durante el proceso y están a cargo de la revisión y aprobación del diseño definitivo. Por otro lado, cuando el "Capstone" busca solucionar un problema o necesidad real, planteada por un ente público o empresa privada, es mayor aún el beneficio pues el estudiantado conoce más de cerca el ejercicio profesional y los procesos industriales y entiende la manera en la que puede aportar desde sus disciplinas. Por otro lado, esta actividad les permite a las industrias entender e interactuar mejor con las instituciones educativas (Shin et al., 2013; Ardis et al., 2013). 
doi: http://dx.doi.org/10.15359/ree.21-2.17

URL: http://www.una.ac.cr/educare

CORREO: educare@una.cr

Tabla 1: Concepciones sobre los proyectos terminal de diseño, tipo "Capstone"

\begin{tabular}{ll}
\hline \multicolumn{1}{c}{ Percepciones sobre "Capstone" } & \multicolumn{1}{c}{ Fuente } \\
\hline $\begin{array}{l}\text { "Capstone" puede ser un curso de un semestre, parte de un curso } \\
\text { de un semestre o un proyecto de investigación como requisito }\end{array}$ & $\begin{array}{l}\text { (Nelson, Cook, \& Raterink, 2013; Oehrtman } \\
\text { et al., 2010; Quinot \& van Tonder, 2014) }\end{array}$ \\
para finalización de formación profesional. & \\
\hline "Capstone" es una herramienta útil para cursos en donde se & \\
requiera la toma de decisiones administrativas y desarrollo de & (Blackford \& Shi, 2015) \\
estrategias gerenciales. & \\
\hline $\begin{array}{l}\text { "Capstone" brinda la posibilidad para que el estudiante se enfrente a } \\
\text { situaciones propias de su ejercicio profesional y puede ser empleado } \\
\text { como evaluación en un examen para otorgar título de maestría. }\end{array}$ & \\
\hline
\end{tabular}

"Capstone" puede ser empleado como un trabajo de investigación en donde el estudiantado se prepara para estudios graduados. Con "Capstone" es posible enfatizar en estrategias metodológicas, (Vellurattil et al., 2014) aspectos éticos y prepara a la persona futura profesional para el manejo o creación de conocimientos avanzados.

El aprendizaje basado en problemas es una forma adecuada de acercar al estudiantado hacia el ejercicio laboral. Este ABP puede ser empleado en "Capstone" enfrentándole a resolver un problema (Stanley \& Marsden, 2013) propio de la industria.

Durante un curso "Capstone" se hace uso de los conocimientos teóricos en casos prácticos en donde el estudiantado debe tomar decisiones referentes a temas ambientales, organizacionales y (Helms \& Whitesell, 2013) de control que atañen a una empresa, resultados que deben ser socializados a sus pares y al empresario.

Como parte de la formación del profesional, se hace necesario que el estudiantado esté en capacidad de integrar la información científica y la práctica para lo cual se requiere que tenga capacidad de realizar una revisión bibliográfica. El "Capstone" puede (Good \& McIntyre, 2015) ser empleado como estrategia pedagógica para mejorar las habilidades de lectura e interpretación en una disciplina.

El equipo de trabajo de evaluación del "Capstone" debe diferenciar entre evaluar los resultados de una investigación y los resultados (Nelson et al., 2013) de la investigación aplicada para resolver un caso.

"Capstone" posibilita que el estudiantado demuestre sus conocimientos y sus habilidades, propicia espacios de divulgación mediante carteles y presentaciones orales y ayuda a cerrar el vacío (Ardis, Hole \& Manfredonia, 2013) existente entre la industria y la academia.

Nota: Autores del artículo. 
La forma como se ha implementado el "Capstone" a través del tiempo varía y, según Stanley \& Marsden (2013), las modalidades o formas pueden encontrarse en cualquiera de las siguientes denominaciones (Stanley \& Marsden, 2013):

- Proyectos finales, tesis e internados

- Reportes escritos de estudios de caso sobre proyectos de la industria

- Presentación de artículos de investigación y críticas de proyectos de investigación y prácticas investigativa

- Prácticas de aprendizaje basado en problemas

Particularmente, el currículo de Ingeniería Civil de la Pontificia Universidad Javeriana Cali tiene como requisito el desarrollo de un trabajo de grado, el cual hasta diciembre de 2015 era realizado de manera individual o en parejas y de manera excepcional en grupos de tres personas. Como resultado del proceso de acreditación ante ABET se determinó desarrollar el trabajo final de grado con la modalidad de "Capstone", lo que generó diferentes opiniones entre el estudiantado, y motivó el desarrollo del estudio de percepción y grado de aceptación del "Capstone" como requisito de grado. Este estudio permitió identificar los cambios a implementarse para las siguientes cohortes.

\section{Problemática abordada}

Existen diferentes modalidades para otorgar el título profesional en las universidades. En algunas se acepta un trabajo de grado, en otras una práctica empresarial o una práctica social, o en otras, un trabajo de investigación; $y$, usualmente, estos trabajos son desarrollados de manera individual o en grupos de máximo tres personas. En otras universidades no se exige trabajo de grado en ninguna modalidad y en su lugar es obligatorio el cumplimiento de cursos y otros requisitos de calidad. En todas las modalidades, el estudiantado recibe su título profesional.

El Ministerio de Educación Nacional y el Concejo Nacional de Acreditación CNA en Colombia aceptan cualquiera de las mencionadas modalidades. Sin embargo, el esquema de acreditación con ABET (Accreditation Board for Engineering and Technology) recomienda que como modalidad de trabajo de grado se ejecute un diseño desarrollado por un grupo de estudiantes en el cual se abarquen, por lo menos, tres áreas de la ingeniería, actividad conocida como "Capstone". La organización académica y administrativa para la implementación del"Capstone"exige una revisión de contenidos de cursos prerrequisito y una preparación de estudiantes y docentes-de tutoría para responder a este requerimiento, por cuanto a pesar de los esfuerzos de las universidades por hacer efectivo el trabajo colaborativo, persisten problemas de tipo aptitudinal, actitudinal y de conocimientos que pueden afectar el rendimiento y el resultado final de este "Capstone". 
doi: http://dx.doi.org/10.15359/ree.21-2.17

URL: http://www.una.ac.cr/educare

CORREO: educare@una.cr

\section{Metodología}

En este estudio, de carácter descriptivo, intervinieron 47 estudiantes de pregrado del curso Fundamentos de Investigación de Ingeniería Civil en la Pontificia Universidad Javeriana Cali, población que hizo parte del régimen de transición entre el sistema de trabajo de grado convencional y el sugerido con "Capstone". Este estudiantado respondió a un instrumento con las siguientes preguntas:

A. Indique las opciones de trabajo final de grado que Ud. conoce de otras universidades: Las opciones de respuesta eran: trabajo de grado, práctica, empresarial, trabajo social, otros.

B. Considera que la modalidad de trabajo de grado "Capstone" es beneficiosa: Las opciones de respuesta eran: Sí, No, Por qué.

C. Dentro de los beneficios que Ud. considera puede tener el trabajo de grado en la modalidad "Capstone" están: Las opciones de respuesta eran: trabajo a semejanza del trabajo en la vida real, ahorro tiempo porque participan más estudiantes atendiendo un mismo objetivo, otro (Por favor especifique).

D. Dentro de las dificultades que puede presentar el trabajo de grado en la modalidad grupal están: Dificultad de coordinar actividades entre los participantes, el equipo de trabajo tiene conocimiento desigual de la temática problema, otro (Por favor especifique).

E. De acuerdo con la información requerida en cada curso (asignatura) responda la consulta correspondiente. Si NO HA VISTO el curso por favor, conteste en todas las casillas que NO HA VISTO EL CURSO. En este caso, se listaron la totalidad de cursos del programa de Ingeniería Civil y para cada uno se debía responder lo siguiente:

- En este curso desarrolló uno o más trabajos de DISEÑO.

- En el curso se emplearon códigos, normas, leyes para realizar el proyecto de DISEÑO

- Se exigió la entrega de un REPORTE ESCRITO

- Se aplicó evaluación de sustentación individual

- Se llevó a cabo sustentación del trabajo de diseño

- Se hizo un análisis de COSTOS de los diseños realizados

- La participación de la totalidad de integrantes fue

- Mi desempeño en el grupo fue

- La composición del grupo (número de estudiantes) era: 0 (para trabajo individual), $2,3,4,5,6$ 
Teniendo en cuenta que el cambio de trabajo de grado a"Capstone" exigía un ajuste académicoadministrativo, durante el desarrollo del curso en el período de transición, se aplicaron unos lineamientos y se hicieron unas recomendaciones para implementación en las siguientes cohortes.

\section{Resultados}

Las universidades tienen diferentes requisitos para grado de sus estudiantes. Al respecto, se observó que más del $50 \%$ de los sujetos encuestados reconoce que existen otras modalidades de grado además del trabajo de grado que se orienta en la Pontificia Universidad Javeriana de Cali ( ver Figura 1).

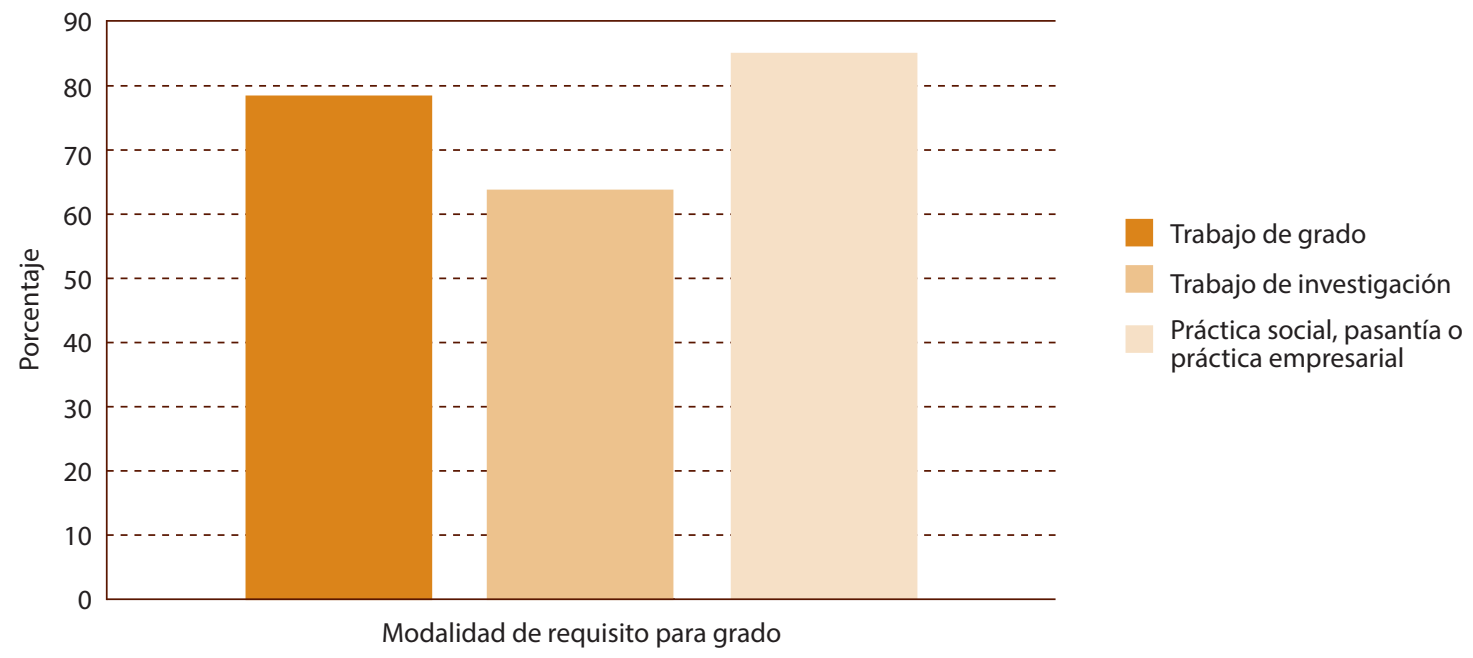

Figura 1: Conocimientos del estudiantado sobre modalidades de trabajo de grado.

Como resultado del cumplimiento de los requerimientos para acreditación ante ABET, fue necesario adaptar la forma de trabajo de grado hacia "Capstone". Al respecto, un $87.2 \%$ considera que esto es una decisión pertinente, que favorece al estudiantado, ya que con esta iniciativa lo enfrenta a su futuro trabajo profesional, a resolver problemas propios de la ingeniería que usualmente resuelve con trabajo colaborativo, con la ventaja de que tendrá a su disposición la dirección de uno o más docentes. Además, un $\mathbf{8 9 . 4 \%}$ de estudiantes considera que los problemas abordados se ajustan a aquellos relacionados con su ejercicio ocupacional y un 31.9\% considera que este tipo de modalidad de grado ahorra tiempo de trabajo y optimiza el tiempo del personal tutor.

En relación con las dificultades que pueden presentarse con la modalidad de trabajo de grado en el esquema de "Capstone", se encontró que el $80.4 \%$ de participantes considera que la coordinación de este tipo de trabajos tiene una mayor dificultad que un trabajo realizado con menor número de personas y un $32.6 \%$ considera que las temáticas abordadas son desconocidas o no han sido tratadas en ninguno de los cursos teóricos. Dentro de la opción 
doi: http://dx.doi.org/10.15359/ree.21-2.17

URL: http://www.una.ac.cr/educare

CORREO: educare@una.cr

de respuesta de otros, se señalaron las siguientes dificultades: "el trabajo recae en una o dos personas", "diferentes asesores complican la definición de objetivos", "no todos los estudiantes tienen la misma carga académica y por lo tanto no todos tienen el mismo tiempo".

En cuanto a las asignaturas en donde el estudiantado ha realizado uno o más trabajos de DISEÑO se encontró que tres de los 18 cursos técnicos-profesionales no incluyen diseño en su desarrollo. No se incluye el curso de ética en este análisis por cuanto en el syllabus de dicho curso no es posible considerar Diseño; sin embargo, en la casuística del curso se pueden considerar dilemas éticos en donde se realiza la revisión de los diseños bajo normas o códigos (ver Tabla 2).

Tabla 2: Porcentaje de cursos con uno o más trabajos de Diseño

De acuerdo con la información requerida en cada curso (asignatura) por favor responda la consulta de cada columna. Si NO HA VISTO el curso, por favor, conteste en todas las casillas que NO HA VISTO EL CURSO.

\begin{tabular}{lcc}
\hline En este curso desarrolló uno o más trabajos de DISEÑO & $\%$ Sí & $\%$ No \\
\hline Diseño Geométrico de Vias I & 80,4 & 19,6 \\
Diseño Geométrico de Vías II & 97,5 & 2,5 \\
Materiales de Ingeniería & 59,6 & 40,4 \\
Métodos Constructivos I & 36,2 & 63,8 \\
Análisis de Estructuras & 93,3 & 6,7 \\
Mecánica de Suelos & 37,0 & 63,0 \\
Mecánica de Fluidos & 51,1 & 48,9 \\
Métodos Constructivos II & 32,1 & 67,9 \\
Fundamentos de Concreto Reforzado & 100,0 & 0,0 \\
Pavimentos & 90,5 & 9,5 \\
Mecánica de Suelos Aplicada & 100,0 & 0,0 \\
Hidráulica de Canales & 97,4 & 2,6 \\
Administración de Obras & 92,3 & 7,7 \\
Diseño de Estructuras de Concreto Reforzado & 100,0 & 0,0 \\
Hidrología & 75,0 & 25,0 \\
Diseño de Fundaciones & 66,7 & 33,3 \\
Proyectos de Construcción & 82,4 & 17,6 \\
Acueducto y Alcantarillado & 100,0 & 0,0 \\
Ética & 24,3 & 75,7 \\
\hline Nota:Autores del aticulo. & &
\end{tabular}

Nota: Autores del artículo. 
doi: http://dx.doi.org/10.15359/ree.21-2.17

URL: http://www.una.ac.cr/educare

En todos los cursos emplearon códigos, normas, o leyes para realizar el proyecto de DISEÑO. Tan solo en cuatro de los 18 cursos técnicos, el estudiantado manifestó un uso de normas en menos de un $50 \%$ de veces (ver Tabla 3 ).

Tabla 3: Porcentaje de cursos en donde emplearon códigos, normas, leyes para realizar el proyecto de Diseño

\begin{tabular}{lrc}
\hline En el curso se emplearon códigos, normas, leyes para realizar el proyecto de DISEÑO & $\%$ Sí & $\%$ No \\
\hline Diseño Geométrico de Vias I & 78,3 & 21,7 \\
Diseño Geométrico de Vías II & 95,0 & 5,0 \\
Materiales de Ingeniería & 63,8 & 36,2 \\
Métodos Constructivos I & 34,0 & 66,0 \\
Análisis de Estructuras & 82,2 & 17,8 \\
Mecánica de Suelos & 43,5 & 56,5 \\
Mecánica de Fluidos & 46,7 & 53,3 \\
Métodos Constructivos II & 35,7 & 64,3 \\
Fundamentos de Concreto Reforzado & 100,0 & 0,0 \\
Pavimentos & 92,9 & 7,1 \\
Mecánica de Suelos Aplicada & 91,1 & 8,9 \\
Hidráulica de Canales & 92,1 & 7,9 \\
Administración de Obras & 70,4 & 29,6 \\
Diseño de Estructuras de Concreto Reforzado & 100,0 & 0,0 \\
Hidrología & 56,8 & 43,2 \\
Diseño de Fundaciones & 60,0 & 40,0 \\
Proyectos de Construcción & 72,2 & 27,8 \\
Acueducto y Alcantarillado & 100,0 & 0,0 \\
Ética & 23,7 & 76,3 \\
\hline
\end{tabular}

Nota: Autores del artículo.

Con respecto a la metodología de evaluación individual se encontró que en todos los cursos se requirió la sustentación individual, pero en seis de los 19 la sustentación individual fue empleada en un porcentaje menor al 50\% (ver Tabla 4). Como se observa en la Tabla 5, prevalece la sustentación colectiva. 
doi: http://dx.doi.org/10.15359/ree.21-2.17

URL: http://www.una.ac.cr/educare

CORREO: educare@una.cr

Tabla 4: Porcentaje de respuestas sobre evaluación de sustentación individual

\begin{tabular}{|c|c|c|}
\hline Se aplicó evaluación de sustentación individual & \% Sí & $\%$ No \\
\hline Diseño Geométrico de Vias I & 60.9 & 39.1 \\
\hline Diseño Geométrico de Vías II & 97.5 & 2.5 \\
\hline Materiales de Ingeniería & 42.6 & 57.4 \\
\hline Métodos Constructivos I & 40.4 & 59.6 \\
\hline Análisis de Estructuras & 57.8 & 42.2 \\
\hline Mecánica de Suelos & 41.3 & 58.7 \\
\hline Mecánica de Fluidos & 42.2 & 57.8 \\
\hline Métodos Constructivos II & 42.9 & 57.1 \\
\hline Fundamentos de Concreto Reforzado & 82.4 & 17.6 \\
\hline Pavimentos & 85.7 & 14.3 \\
\hline Mecánica de Suelos Aplicada & 77.8 & 22.2 \\
\hline Hidráulica de Canales & 84.2 & 15.8 \\
\hline Administración de Obras & 81.5 & 18.5 \\
\hline Diseño de Estructuras de Concreto Reforzado & 88.9 & 11.1 \\
\hline Hidrología & 50.0 & 50.0 \\
\hline Diseño de Fundaciones & 50.0 & 50.0 \\
\hline Proyectos de Construcción & 77.8 & 22.2 \\
\hline Acueducto y Alcantarillado & 78.3 & 21.7 \\
\hline Ética & 28.9 & 71.1 \\
\hline
\end{tabular}

Nota: Autores del artículo.

En relación con la sustentación del trabajo de diseño, lo cual se considera como una actividad evaluativa para el grupo de trabajo, se observa que en todos los cursos se emplea esta estrategia. Sin embargo, en cinco de las 19 asignaturas la sustentación colectiva tuvo un nivel de participación menor al 50\% (ver Tabla 5).

10 María Fernanda Serrano-Guzmán, Diego Darío Pérez-Ruiz, Luz Marina Torrado-Gómez y Norma Cristina Solarte-Vanegas 
doi: http://dx.doi.org/10.15359/ree.21-2.17

URL: http://www.una.ac.cr/educare

CORREO: educare@una.cr

Tabla 5: Porcentaje de respuestas relacionadas con sustentación grupal del trabajo de diseño

\begin{tabular}{lcc}
\hline Se llevó a cabo sustentación del trabajo de diseño & $\%$ Sí & $\%$ No \\
\hline Diseño Geométrico de Vias I & 63,0 & 37,0 \\
\hline Diseño Geométrico de Vías II & 100,0 & 0,0 \\
Materiales de Ingeniería & 46,8 & 53,2 \\
\hline Métodos Constructivos I & 31,9 & 68,1 \\
Análisis de Estructuras & 62,2 & 37,8 \\
Mecánica de Suelos & 28,3 & 71,7 \\
Mecánica de Fluidos & 48,9 & 51,1 \\
Métodos Constructivos II & 32,1 & 67,9 \\
Fundamentos de Concreto Reforzado & 88,2 & 11,8 \\
Pavimentos & 83,3 & 16,7 \\
Mecánica de Suelos Aplicada & 86,7 & 13,3 \\
Hidráulica de Canales & 94,7 & 5,3 \\
Administración de Obras & 85,2 & 14,8 \\
Diseño de Estructuras de Concreto Reforzado & 100,0 & 0,0 \\
Hidrología & 52,3 & 47,7 \\
Diseño de Fundaciones & 70,0 & 30,0 \\
Proyectos de Construcción & 72,2 & 27,8 \\
Acueducto y Alcantarillado & 78,3 & 21,7 \\
\hline Ética & 21,1 & 78,9 \\
\hline Ma: Autores & & \\
\hline
\end{tabular}

Nota: Autores de artículo.

La consideración de los costos en los diseños realizados fue abordada en cuatro de las 19 asignaturas del ciclo profesional. En el resto de asignaturas, menos del $50 \%$ de los sujetos encuestados realizó análisis de costos a los diseños o proyectos realizados en los distintos cursos (ver Tabla 6). 
doi: http://dx.doi.org/10.15359/ree.21-2.17

URL: http://www.una.ac.cr/educare

CORREO: educare@una.cr

Tabla 6: Porcentaje de respuestas relacionadas con asignaturas en donde se incluyeron análisis de costos en los diseños realizados

\begin{tabular}{l|ll}
\hline Se hizo un análisis de COSTOS de los diseños realizados & $\%$ Sí & $\%$ No \\
\hline Diseño Geométrico de Vías I & 8.7 & 91.3 \\
\hline Diseño Geométrico de Vías II & 17.5 & 82.5 \\
\hline Materiales de Ingeniería & 19.1 & 80.9 \\
\hline Métodos Constructivos I & 29.8 & 70.2 \\
\hline Análisis de Estructuras & 15.6 & 84.4 \\
\hline Mecánica de Suelos & 15.2 & 84.8 \\
\hline Mecánica de Fluidos & 22.2 & 77.8 \\
\hline Métodos Constructivos II & 21.4 & 78.6 \\
\hline Fundamentos de Concreto Reforzado & 23.5 & 76.5 \\
\hline Pavimentos & 23.8 & 76.2 \\
\hline Mecánica de Suelos Aplicada & 75.6 & 24.4 \\
\hline Hidráulica de Canales & 28.9 & 71.1 \\
\hline Administración de Obras & 85.2 & 14.8 \\
\hline Diseño de Estructuras de Concreto Reforzado & 55.6 & 44.4 \\
\hline Hidrología & 25.0 & 75.0 \\
\hline Diseño de Fundaciones & 20.0 & 80.0 \\
\hline Proyectos de Construcción & 72.2 & 27.8 \\
\hline Acueducto y Alcantarillado & 39.1 & 60.9 \\
\hline Ética & 18.4 & 81.6 \\
\hline Pa: Autors & & \\
\hline
\end{tabular}

Nota: Autores de artículo.

Con respecto a la actitud de trabajo en grupo, el $86.20 \%$ del estudiantado en promedio manifiesta que la participación del grupo fue buena, frente al $12.50 \%$ y $1.3 \%$ de participación regular y mala, respectivamente (ver Tabla 7). 
doi: http://dx.doi.org/10.15359/ree.21-2.17

URL: http://www.una.ac.cr/educare

CORREO: educare@una.cr

Tabla 7: Porcentaje de respuestas relacionadas con actitud de trabajo de los integrantes del grupo de Diseño

\begin{tabular}{lccc}
\hline La participación de todos los integrantes fue & \% Buena & \% Regular & $\%$ Mala \\
\hline Diseño Geométrico de Vías I & 69.20 & 30.80 & 0 \\
Diseño Geométrico de Vías II & 65.80 & 23.70 & 10.5 \\
Materiales de Ingeniería & 70.00 & 26.70 & 3.3 \\
Métodos Constructivos I & 100.00 & 0.00 & 0 \\
Análisis de Estructuras & 82.50 & 15.00 & 2.5 \\
Mecánica de Suelos & 87.00 & 13.00 & 0 \\
Mecánica de Fluidos & 80.80 & 15.40 & 3.8 \\
Métodos Constructivos II & 92.30 & 7.70 & 0 \\
Fundamentos de Concreto Reforzado & 88.20 & 11.80 & 0 \\
Pavimentos & 83.30 & 16.70 & 0 \\
Mecánica de Suelos Aplicada & 85.70 & 11.90 & 2.4 \\
Hidráulica de Canales & 94.40 & 5.60 & 0 \\
Administración de Obras & 88.50 & 11.50 & 0 \\
Diseño de Estructuras de Concreto Reforzado & 80.00 & 20.00 & 0 \\
Hidrología & 88.90 & 8.30 & 2.8 \\
Diseño de Fundaciones & 100.00 & 0.00 & 0 \\
Proyectos de Construcción & 93.80 & 6.30 & 0 \\
Acueducto y Alcantarillado & 87.00 & 13.00 & 0 \\
Ética & 100.00 & 0.00 & 0 \\
\hline
\end{tabular}

Nota: Autores de artículo.

Con respecto a la actitud personal ante el trabajo colectivo, el $95.22 \%$ de estudiantes en promedio manifiestan que la participación personal fue buena, frente al $4.2 \%$ y $0.6 \%$ de participación regular y mala, respectivamente (ver Tabla 8). 
doi: http://dx.doi.org/10.15359/ree.21-2.17

URL: http://www.una.ac.cr/educare

CORREO: educare@una.cr

Tabla 8: Porcentaje de respuestas relacionadas con actitud de trabajo individual en el trabajo de Diseño

\begin{tabular}{lccc}
\hline Mi desempeño en el grupo fue & \% Bueno & \% Regular & $\%$ Malo \\
\hline Diseño Geométrico de Vías I & 91.3 & 8.7 & 0 \\
Diseño Geométrico de Vías II & 89.7 & 2.6 & 7.7 \\
Materiales de Ingeniería & 88.9 & 11.1 & 0 \\
Métodos Constructivos I & 97.7 & 2.3 & 0 \\
Análisis de Estructuras & 95.3 & 4.7 & 0 \\
Mecánica de Suelos & 100 & 0 & 0 \\
Mecánica de Fluidos & 97.5 & 2.5 & 0 \\
Métodos Constructivos II & 91.7 & 8.3 & 0 \\
Fundamentos de Concreto Reforzado & 100 & 0 & 0 \\
Pavimentos & 90 & 10 & 0 \\
Mecánica de Suelos Aplicada & 95.5 & 4.5 & 0 \\
Hidráulica de Canales & 100 & 0 & 0 \\
Administración de Obras & 89.7 & 6.9 & 3.4 \\
Diseño de Estructuras de Concreto Reforzado & 100 & 0 & 0 \\
Hidrología & 95.2 & 4.8 & 0 \\
Diseño de Fundaciones & 100 & 0 & 0 \\
Proyectos de Construcción & 94.1 & 5.9 & 0 \\
Acueducto y Alcantarillado & 95.8 & 4.2 & 0 \\
Ética & 96.9 & 3.1 & 0 \\
\hline Mota: Autores & & 0 \\
\hline
\end{tabular}

Nota: Autores de artículo.

Con respecto a la composición de los grupos de trabajo, se observa una mayor inclinación por los grupos de tres personas (33\% en promedio) seguido por el trabajo individual con una preferencia en promedio del $21 \%$. Los grupos de trabajo de más de 4 personas según esta encuesta no son comunes en los proyectos de aula (ver Tabla 9). 
doi: http://dx.doi.org/10.15359/ree.21-2.17

URL: http://www.una.ac.cr/educare

CORREO: educare@una.cr

Tabla 9: Porcentaje de respuestas relacionadas con composición de los grupos de trabajo en el proyecto de Diseño

\begin{tabular}{lcccccc}
\hline $\begin{array}{l}\text { Cuál era el número de estudiantes que } \\
\text { participaron en el desarrollo del proyecto }\end{array}$ & 1 & 2 & 3 & 4 & 5 & 6 \\
\hline Diseño Geométrico de Vías I & 20 & 13.3 & 31.1 & 31.1 & 4.4 & 0 \\
Diseño Geométrico de Vías II & 4.9 & 17.1 & 51.2 & 24.4 & 2.4 & 0 \\
Materiales de Ingeniería & 37.2 & 2.3 & 14 & 30.2 & 16.3 & 0 \\
Métodos Constructivos & 56.1 & 22 & 19.5 & 2.4 & 0 & 0 \\
Análisis de Estructuras & 2.3 & 46.5 & 30.2 & 20.9 & 0 & 0 \\
Mecánica de Suelos & 46.5 & 7 & 20.9 & 16.3 & 9.3 & 0 \\
Mecánica de Fluidos & 41.5 & 9.8 & 19.5 & 22 & 2.4 & 4.9 \\
Métodos Constructivos II & 51.7 & 6.9 & 17.2 & 10.3 & 13.8 & 0 \\
Fundamentos de Concreto Reforzado & 2.7 & 40.5 & 13.5 & 18.9 & 18.9 & 5.4 \\
Pavimentos & 25.6 & 16.3 & 32.6 & 20.9 & 4.7 & 0 \\
Mecánica de Suelos Aplicada & 0 & 6.7 & 24.4 & 48.9 & 17.8 & 2.2 \\
Hidráulica de Canales & 2.4 & 14.6 & 70.7 & 7.3 & 4.9 & 0 \\
Administración de Obras & 3.8 & 30.8 & 61.5 & 3.8 & 0 & 0 \\
Diseño de Estructuras de Concreto & 10 & 30 & 30 & 10 & 20 & 0 \\
Reforzado & & & & & & \\
Hidrología & 7.3 & 12.2 & 26.8 & 39 & 12.2 & 2.4 \\
Diseño de Fundaciones & 70 & 10 & 20 & 0 & 0 & 0 \\
Proyectos de Construcción & 0 & 11.1 & 77.8 & 5.6 & 5.6 & 0 \\
Acueducto y Alcantarillado & 4 & 8 & 40 & 44 & 4 & 0 \\
\hline
\end{tabular}

Nota: Autores de artículo.

\section{Discusión}

Como se mencionó, el objetivo de este estudio era medir la percepción de la población de estudiantes del régimen de transición entre el trabajo de grado convencional y la modalidad de "Capstone" (Diseño I y Diseño II) y presentar las consideraciones correspondientes, de tipo académico y administrativo, para que el desarrollo del "Capstone" sea viable en Ingeniería Civil. 
doi: http://dx.doi.org/10.15359/ree.21-2.17

URL: http://www.una.ac.cr/educare

CORREO: educare@una.cr

Por lo anterior, a continuación, se discuten los principales hallazgos y planeamientos tomando en consideración dos apartados: las reflexiones relacionadas con la percepción del estudiantado y de las consideraciones académico-administrativas para la implementación.

\section{Reflexiones con relación a la percepción del estudiante}

La población que fue seleccionada en este estudio correspondió a la cohorte del régimen de transición entre el curso Fundamentos de Investigación, que actualmente es Diseño l, que se adelanta en un semestre como requisito para Trabajo de Grado curso que en la actualidad es Diseño Il y que se debe cursar en el semestre siguiente. Desde esa perspectiva, entonces, se deberá incluir una modalidad de grado adicional a las existentes (Figura 1) que para afectos del programa de acreditación por ABET es reconocido como "Capstone".

Para el caso particular de la Pontificia Universidad Javeriana, en la mayoría de las asignaturas del currículo se contemplan actividades de Diseño. Esto facilitó el cambio de un esquema (Trabajo de Grado) por otro ("Capstone") por cuanto el estudiantado está familiarizado con el tipo de entregables (informe) para cada curso y por ende para cada tópico y con los planos de construcción correspondientes. Así mismo, en la mayoría de asignaturas se propende por el trabajo en grupo. En general, la mayoría del estudiantado (87.2\%) considera favorable la implementación del "Capstone" porque les se enfrenta a problemáticas similares a las de la vida real que pueden ser atendidas mediante el trabajo en equipo en menos tiempo.

Lo que resulta convenienteempezar a trabajar al interior del programa es que la conformación de estos grupos se haga no por libre selección del estudiante, sino que sea producto del azar, lo cual se aproxima más a lo que ocurre en el campo laboral.Y, que además de la sustentación grupal, se emplee la estrategia de sustentación individual, que no es tan difundida en la actualidad y que es una herramienta útil para el desarrollo de competencias de comunicación oral.

Ahora bien, aunque la entrevista (diario de campo) no fue empleada en este estudio, es importante mencionar que los diálogos espontáneos con el estudiantado permitieron identificar los principales temores de estos acerca de las posibles dificultades del desarrollo del trabajo en grupo ante las diferencias en las competencias de sus pares y del impacto que esto tendría en la calificación del curso.

\section{Reflexiones académico-administrativas para la implementación del "Capstone”}

Un curso de "Capstone" permite que el estudiante se enfrente o haga uso de lo aprendido. Por lo tanto, este tipo de estrategia pedagógica es positiva para los estudiantes. Además, por parte de la industria se ha encontrado que aquellos estudiantes que intervienen en este tipo de proyectos verdaderamente mejoran su desempeño profesional y se posicionan laboralmente más rápido e inclusive con mejores salarios que aquellos que no realizaron un proyecto "Capstone" 
(Gruenther et al., 2009), por cuanto le permite al estudiante y futuro profesional continuar su proceso de aprendizaje mientras resuelve un problema (Quinot \& van Tonder, 2014).

Con el curso "Capstone" se espera que los estudiantes comprendan y realicen diseños siguiendo requerimientos, leyes, códigos y normas. Para ello, se genera el espacio propicio para el aprendizaje independiente y para el trabajo en grupo con un programa de respuestas o entregables por metas u objetivos (Cooper, Fulton \& Homan, 2015). Sin embargo, se observa que los integrantes de los grupos de trabajo deben haber concluido el ciclo de diseño en las distintas áreas para poder apoyar las actividades de diseño que den solución a la problemática abordada; es decir, la implementación del "Capstone" exige haber cursado y aprobado unos cursos de diseño de alto nivel, por lo general ofrecidos en secuencia (Agboola, et al., 2012).

Adicionalmente, previo a "Capstone", debe darse un curso en el que se entrene al estudiantado en la elaboración de la revisión bibliográfica, en el desarrollo de habilidades de comunicación escrita y en los adecuados procesos de citación y referenciación para evitar los problemas de plagio (Agboola, et al., 2012; Helms \& Whitesell, 2013). Cabe acotar que esta actividad se realiza de manera individual e independiente (Osman, Jaafar, Wan Badaruzzaman, \& Rahmat, 2012) y que resulta pertinente generar estrategias para desarrollar las habilidades de síntesis mediante el registro del diario ocurrir del trabajo del grupo, a manera de bitácora (Agboola et al., 2012; Osman et al., 2012).

Otro aspecto a considerar es la duración del proyecto que debe ser máximo de dos semestres, incluyendo el curso previo a "Capstone" (Diseño l) y el "Capstone" (Diseño II) (Agboola et al., 2012; Osman et al., 2012). Le corresponde al profesorado encargado del curso de Diseño I establecer, desde el comienzo, los tiempos de ejecución de las actividades (Agboola et al., 2012).

Ahora bien, es recomendable que en el segundo semestre de "Capstone" (Diseño II), el equipo de trabajo presente un reporte de los diseños realizados a un jurado (Agboola et al., 2012). Este reporte (informe) se redacta en conjunto con todos los integrantes del grupo (Osman et al., 2012) y se compone de un informe técnico, planos de construcción, resultados de simulaciones por computador si las hubo y preparación de presupuestos (Osman et al., 2012).

Otra realidad es que el uso del aprendizaje basado en problemas (ABP) como estrategia pedagógica en el currículo prepara al estudiantado para el "Capstone", por cuanto con el ABP se hace uso de un problema general y se orienta al estudiantado a su solución buscando con ello una identidad profesional.

En cuanto a la composición de los equipos de trabajo, se recomienda como máximo cinco estudiantes (Stanley \& Marsden, 2013). Estudios previos revelan que la ventaja del trabajo en grupo es que permite un aprendizaje más profundo, se logran mejores resultados en calificaciones, permite el desarrollo de habilidades sociales y valores cívicos, ayuda a estructurar el pensamiento, promueve el crecimiento personal y desarrolla actitudes positivas frente al aprendizaje autónomo (Lubbers, 2011), además de que se aprende a trabajar en equipos interdisciplinarios (Odora, 2015). 
doi: http://dx.doi.org/10.15359/ree.21-2.17

URL: http://www.una.ac.cr/educare

CORREO: educare@una.cr

Estudiantes de "Capstone" necesitan de la asesoría de personal experto para comprender y contribuir en el mercado laboral y responder a las necesidades de la industria (Odora, 2015). Además, cuando el proyecto "Capstone" se realiza multidisciplinariamente se aumentan las expectativas tanto de estudiantes como de docentes-tutores y se incrementan las habilidades personales y las técnicas propias de cada disciplina y se complementan con las de sus colaboradores (Cooper et al., 2015). Existen evidencias, inclusive, de trabajos interdisciplinarios e interinstitucionales con los cuales el ejercicio de "Capstone" mejoró las habilidades en general de sus participantes (Ardis et al., 2013).

El desarrollo de "Capstone" como opción de modalidad de grado para estudiantes de pregrado es una realidad en diferentes instituciones educativas. Los referentes consultados desarrollan el "Capstone" en uno o dos semestres (Oehrtman et al., 2010) y se emplean diferentes modalidades buscando que la persona futura profesional realice un ejercicio práctico muy cercano al ejercicio laboral. Sin embargo, se observa que se deben reforzar las cualidades del trabajo colaborativo durante el tiempo de formación para garantizar la meta global partiendo del cumplimiento de metas individuales.

La actitud del estudiantado frente al trabajo en grupo debe ser considerada por el equipo de docentes-tutores. Aunque es común que los grupos de trabajo se organicen de manera espontánea y voluntaria, es una realidad que durante el ejercicio laboral el futuro ingeniero o ingeniera se enfrenta a colegas de trabajo que en ocasiones no tienen su mismo nivel de desempeño, pero, aun así, responden a las necesidades del ente empleador o de lo contrario pierden su empleo. Con esta mentalidad, algunos grupos de estudiantes han manejado los rendimientos al interior del equipo de trabajo, mediante la presión hacia quienes no responden, delegándoles tareas que no comprometan el resultado final, y en caso extremo, reportando al director o directora los inconvenientes, a la espera de que sea el personal docente quien organice nuevamente al grupo y tome la decisión de exclusión de integrantes que no estén aportando.

Para que el "Capstone" sea efectivo, la institución que lo implemente debe establecer lineamientos académicos y administrativos que permitan que los proyectos se ejecuten con el saber del estudiantado, lo cual exige haber cursado y aprobado un mínimo número de cursos profesionales.

Dentro de los criterios administrativos se señalan:

- En relación con la labor docente. Debe destinarse por lo menos dos horas de dedicación al director o directora principal y, por lo menos, una hora de atención a cada asesor o asesora. Adicionalmente, para el equipo de asesoría, debe contemplarse una hora de atención grupal en sesión simultánea asesorías y estudiantes, en la que se socialicen los avances de los trabajos adelantados de manera individual. Esto representa un tiempo de dedicación recomendado de tres horas para quien dirige y por lo menos de dos horas para cada persona asesora, por cada proyecto "Capstone". 
- El requerimiento de una hora común para reuniones grupales. Esto implica que en el horario del semestre regular debe programarse esta franja de tiempo disponible, tanto para estudiantes como para docentes con el fin que se dé la coincidencia.

- En cuanto al número máximo de proyectos "Capstone" que puede dirigir una persona docente. Debe considerarse que un curso regular de clase, con intensidad horaria de 4 horas, implica 4 horas de preparación de clases y evaluación y 2 horas de atención de estudiantes por cada docente. Es decir, un curso, con un solo grupo representa 10 horas de clase y para dos cursos, un solo grupo, 20 horas de clase. Si este docente tiene 3 proyectos "Capstone", implicaría que por lo menos requeriría 9 horas semanales, para un total de 29 horas de solo docencia. Como se observa, si un docente dirige tres proyectos "Capstone" no tendrá tiempo para realizar investigación ni para adelantar labores administrativas.

- Según este análisis, el número máximo de proyectos "Capstone" que puede atender una persona docente es de 3 , bien sea como director o directora principal o como personal asesor. La anterior consideración deja entrever que se requerirá la contratación de profesorado externo para atender los cursos regulares o el "Capstone".

Sobre los criterios académicos, deben definirse:

- Contenido de Diseño I, curso previo (requisito) en el "Capstone". Esto por cuanto se hace necesario que se defina la problemática a abordar, se fortalezcan las competencias de comunicación oral y escrita y se conozcan y respeten los plazos de ejecución de los proyectos, situación fundamental para definir el alcance del entregable final (Diseño II). Justamente, el requerimiento de cumplimiento en los tiempos de ejecución de los proyectos se señala como una de las barreras académica para la implementación del "Capstone" (Nelson et al., 2013). Otra barrera encontrada es que el personal docente encargado del curso prerrequisito de "Capstone" debe ser el mismo de "Capstone" (Diseño II) a menos que se desarrolle una estrategia de comunicación entre docentes participantes de modo que se garantice el conocimiento del trabajo adelantado por los distintos equipos.

- Mecanismos de evaluación relacionados con porcentajes sobre el trabajo individual ygrupal y sobre las habilidades esperadas a nivel individual y grupal. Además del seguimiento del trabajo grupal es una labor de personal docente-tutor supervisar las entregas parciales de cada integrante del grupo, de manera que se pueda detectar, tempranamente, a quien pudiera afectar la entrega del proyecto en la fecha señalada. En cuanto a la sustentación grupal, le corresponde al personal docente guía y al personal tutor evaluar conjuntamente la competencia de comunicación oral individual y grupal, lo cual se facilita con el uso de una rúbrica que estandarice los criterios al momento de hacer la evaluación. 
doi: http://dx.doi.org/10.15359/ree.21-2.17

URL: http://www.una.ac.cr/educare

CORREO: educare@una.cr

- Además, con el fin de contrarrestar las experiencias negativas por falta de motivación (desánimo o desinterés) estudiantil por el trabajo que se realiza y justamente para controlar estos inconvenientes actitudinales se debe plantear, además de la evaluación del trabajo en grupo y la evaluación individual por la parte docente, una evaluación por parte de cada integrante a sus pares (heteroevaluación) (Lubbers, 2011). Se ha demostrado que este tipo de evaluación permite otorgar un real reconocimiento a aquellos o aquellas estudiantes que trabajan con responsabilidad y entrega.

- En relación con la competencia escrita, es recomendable que, a lo largo del proyecto, el estudiantado realice entregas parciales de manera que se revise oportunamente el documento antes de editar el documento final. Al respecto, las modalidades de redacción de cada personal docente-tutor varían, por lo cual es conveniente definir internamente la conducta de entrega del documento, y generar unos controles de custodia del documento revisado, esto con el fin de propiciar un trabajo colaborativo tácito entre docentes-tutores de manera que no se rechacen las decisiones que previamente se han tomado. Esto se logra, si se organizan reuniones semanales, en donde se socializan avances y se hacen acuerdos registrados en actas levantadas en cada sesión, para la revisión de los documentos.

- A nivel curricular, es pertinente que en aquellos cursos en los cuales se adelanten proyectos de diseño, se incorporen requisitos de uso de normas, leyes y códigos; se elaboren presupuestos para que se tomen decisiones técnico-económicas; se soliciten reportes escritos con normas de publicación de altos estándares de calidad y se promuevan espacios para la divulgación; acciones con las cuales se logra el entrenamiento requerido del estudiantado. Al respecto, se sugiere que las presentaciones de los trabajos grupales se hagan extensivas a toda la comunidad académica y que se generen los medios para que estas presentaciones sean evaluadas, con ciertos criterios de calidad, por asistentes.

- Se deben fortalecer los principios del trabajo colaborativo para que el estudiantado aprenda a aprender mediante el trabajo en equipo. Esto implica tener en cuenta las características personales que inciden en el desempeño del individuoy las características del grupo (Blackford \& Shi, 2015).

- Se deben definir rúbricas de aceptación del documento final tanto para la directora o el director del "Capstone" como para el personal asesor. Puede ser posible que se genere un sistema de apoyo por intranet o en aplicaciones por web de manera que la información pueda ser revisada y la trazabilidad sea almacenada en una página o portal diseñado para tal fin. 


\section{Conclusiones}

La modalidad de "Capstone" como estrategia de trabajo de grado permite el acercamiento del estudiantado hacia el ejercicio profesional. En general, su percepción es favorable respecto a la implementación de "Capstone" (Diseño I y Diseño II), además que se posee la certeza que este requisito de grado tiene que ser culminado en máximo dos semestres, situación que no ocurría en el proyecto de grado convencional en donde, dependiendo del tema, el estudiantado pudiera tardarse mucho más.

La decisión institucional de acogerse a esta modalidad exige organización administrativa y académica al interior, ya que el tiempo de dirección de los proyectos debe ser incorporado en la labor docente y deben establecerse requisitos mínimos para que el estudiantado acceda al "Capstone", así como también, considerar la posibilidad de contratación de personal externo para la dirección de los trabajos, cuando esto fuere necesario.

Además, deben programarse los horarios de personal docente-tutor de manera tal que se den tiempos de convergencia entre estos grupos y estudiantes.

Se recomienda también que se definan rúbricas para el control de documentos y para el recibo del documento final.

\section{Referencias}

Agboola, O. P., Hashemipour, M., Egelioglu, F., Atikol, U., \& Hacisevki, H. (2012). Assessing a decade old capstone senior projects through ABET accreditation program outcomes. Procedia - Social and Behavioral Sciences, 47, 120-125. doi: https://doi.org/10.1016/j. sbspro.2012.06.624

Ardis, M., Hole, E., \& Manfredonia, J. (2013). Creating a marketplace for multidisciplinary multiuniversity systems engineering capstone projects. Procedia Computer Science, 16, 10361042. doi: https://doi.org/10.1016/j.procs.2013.01.109

Blackford, B., \& Shi, T. (2015). The relationship between business simulations in capstone management courses and standardized test scores. The International Journal of Management Education, 13(1), 84-94. doi: https://doi.org/10.1016/j.ijme.2015.01.005

Cooper, C., Fulton, J., \& Homan, J. (2015). A multi-spectrum framework for characterizing interdisciplinary capstone design experiences. Procedia Computer Science, 44, 699-707. doi: https://doi.org/10.1016/j.procs.2015.03.018

Good, D. J., \& Mclntyre, C. (2015). Use of journal clubs within senior capstone courses: Analysis of perceived gains in reviewing scientific literature. Journal of Nutrition Education and Behavior, 47(5), 477-479. doi: https://doi.org/10.1016/j.jneb.2015.05.003 
doi: http://dx.doi.org/10.15359/ree.21-2.17

URL: http://www.una.ac.cr/educare

CORREO: educare@una.cr

Gruenther, K., Bailey, R., Wilson, J., Plucker, C., \& Hashmi, H. (2009). The influence of prior industry experience and multidisciplinary teamwork on student design learning in a capstone design course. Design Studies, 30(6), 721-736. doi: https://doi.org/10.1016/j. destud.2009.06.001

Helms, M. M., \&Whitesell, M. (2013). Transitioning to the embedded librarian model and improving the senior capstone business strategy course. The Journal of Academic Librarianship, 39(5), 401-413. doi: https://doi.org/10.1016/j.acalib.2013.03.015

Lubbers, C. A. (2011). An assessment of predictors of student peer evaluations of team work in the capstone campaigns course. Public Relations Review, 37, 492-498. doi: https://doi. org/10.1016/j.pubrev.2011.09.013

Nelson, J. M., Cook, P. F., \& Raterink, G. (2013). The evolution of a doctor of nursing practice capstone process: Programmatic revisions to improve the quality of student projects, 29(6), 370-380. doi: https://doi.org/10.1016/j.profnurs.2012.05.018

Odora, R. J. (2015). Integrating product design and entrepreneurship education: A stimulant for enterprising design and engineering students in South Africa. Procedia Technology, 20, 276-283. doi: https://doi.org/10.1016/j.protcy.2015.07.044

Oehrtman, S. J., Smolen, D., Hoblet, K. \&, Phillips, K. A. (2010). The comprehensive examination: A viable master's of science in nursing capstone course. Journal of Professional Nursing, 26(6), 360-365. doi: https://doi.org/10.1016/j.profnurs.2010.08.003

Osman, S. A., Jaafar, O., Wan Badaruzzaman, W. H. W. \&, Rahmat, R. A. (2012). The course outcomes (COs) evaluation for civil engineering design course. Procedia - Social and Behavioral Sciences, 60, 103-111. doi: https://doi.org/10.1016/j.sbspro.2012.09.354

Quinot, G., \& van Tonder, S.P. (2014). The potential of capstone learning experiences in addressing perceived shortcomings in LLB training in South Africa. PER: Potchefstroomse Elektroniese Regsblad, 17(4), 1350-1390. doi: https://doi.org/10.4314/pelj.v17i4.05

Shin, Y.-S., Lee K-W., Ahn, J-S. \& Jung, J-W. (2013). Development of Internship \& Capstone Design Integrated Program for University-Industry Collaboration. Procedia - Social and Behavioral Sciences, 102, 386-391. doi: https://doi.org/10.1016/j.sbspro.2013.10.753

Stanley, T., \& Marsden, S. (2013). Accountancy capstone: Enhancing integration and professional identity. Journal of Accounting Education, 31(4), 363-382. doi: https://doi.org/10.1016/j. jaccedu.2013.08.002

Vellurattil, R. P., Puglisi, M. P., Johnson, C. L. \&, Slonek, J. (2014). Introduction of a capstone research program in a new college of pharmacy: Student perceptions. Currents in Pharmacy Teaching and Learning, 6(3), 429-436. https://doi.org/10.1016/j.cptl.2014.02.008 